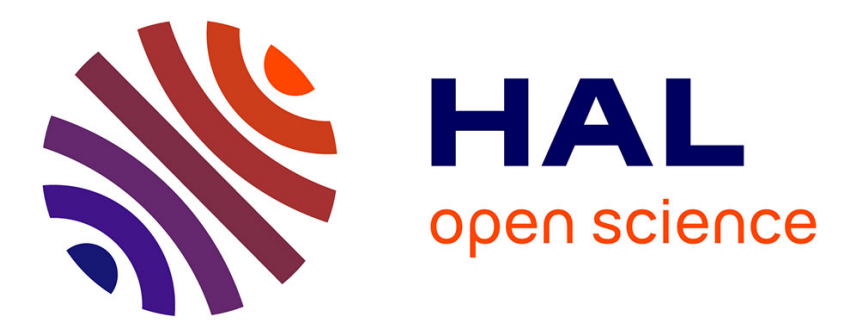

\title{
Deterministic Method for Automatic Visual Grading of Seed Food Products
}

Pierre Dubosclard, Stanislas Larnier, Hubert Konik, Ariane Herbulot, Michel Devy

\section{- To cite this version:}

Pierre Dubosclard, Stanislas Larnier, Hubert Konik, Ariane Herbulot, Michel Devy. Deterministic Method for Automatic Visual Grading of Seed Food Products. 4th International Conference on Pattern Recognition Applications and Methods, Jan 2015, Lisbon, Portugal. 10.5220/0005188102120217. hal-01355080

\author{
HAL Id: hal-01355080 \\ https://hal.science/hal-01355080
}

Submitted on 22 Aug 2016

HAL is a multi-disciplinary open access archive for the deposit and dissemination of scientific research documents, whether they are published or not. The documents may come from teaching and research institutions in France or abroad, or from public or private research centers.
L'archive ouverte pluridisciplinaire HAL, est destinée au dépôt et à la diffusion de documents scientifiques de niveau recherche, publiés ou non, émanant des établissements d'enseignement et de recherche français ou étrangers, des laboratoires publics ou privés. 


\title{
Deterministic method for automatic visual grading of seed food products
}

\author{
Pierre Dubosclard ${ }^{1,2}$, Stanislas Larnier ${ }^{1,2}$, Hubert Konik $^{3}$, Ariane Herbulot ${ }^{1,2}$ and Michel Devy ${ }^{1,2}$ \\ ${ }^{1}$ CNRS, LAAS, 7 avenue du colonel Roche, F-31400 Toulouse, France \\ ${ }^{2}$ Univ de Toulouse, UPS, LAAS, F-31400 Toulouse, France \\ ${ }^{3}$ Laboratoire Hubert Curien, Saint-Etienne \\ \{pierre.dubosclard, stanislas.larnier, ariane.herbulot, michel.devy\}@laas.fr, hubert.konik@univ-st-etienne.fr
}

\begin{abstract}
Keywords: Image processing, image segmentation, shape learning, active contours, visual grading.
Abstract: $\quad$ This paper presents a deterministic method for automatic visual grading, designed to solve the industrial problem of evaluation of seed lots. The sample is thrown in bulk onto a tray placed in a chamber for acquiring color image. An image processing method had been developed to separate and characterize each seed. Shape learning is performed on isolated seeds. The collected information is used for the segmentation. A first step is made based on simple criteria such as regions, edges and normals to the boundary. Then, an active contour with shape prior is performed to improve the results.
\end{abstract}

\section{INTRODUCTION}

In agriculture, the global grain harvest reached several billion tons each year. Seed producers exchange their crops at a price determined by the quality of their production. This assessment, called grading, is performed for each set on a representative sample. The difficulty of this assessment is to fully characterize the sample. To do so, it is necessary to qualify each of its elements. Historically, this has been performed manually by an operator. This method is exposed to various problems and the results can vary from one operator to another.

Alpha MOS company develops systems for quality control of food products. It proposes a visual sensory system to provide an alternative to human evaluation. The assessment should be simple to implement and at least as fast as the human evaluation.

The seed samples are presented in bulk, without any arrangement, but they are spread over a tray in such way that there is no overlapping between the objects to avoid occlusion (Figure 1). The evaluation by the instrument is composed of three steps. The first step is the acquisition: the operator places the samples on the tray in the instrument and takes an image. Then the detection step consists in finding each object in the image, to finally classify them in different quality classes regarding several criteria (shape, color, spot) during the classification step.

The quality of the sample can then be deduced from the result of the classification. The detection
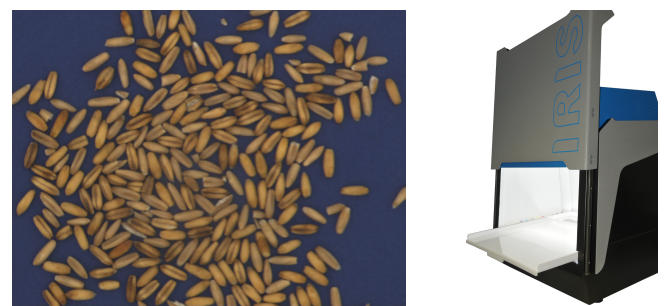

Figure 1: Wheat seeds in bulk and acquisition system.

step is the main difficulty. It is necessary to develop a segmentation method to isolate each object under the following constraints: the number of seeds is unknown (an approximate estimation can be done); the objects have quite generical geometric and chromatic features; they are randomly placed, without arrangement and with no overlapping.

In Section 2, a state of the art around the visual grading problem is presented. Section 3 describes the data acquisition system. Our approach needs prior shape information. The shape learning on binarized images is explain in Section 4. The segmentation approach to detect the seeds is provided in Section 5. Numerical results are presented in Section 6.

\section{STATE OF THE ART}

Several studies have been conducted on the cereal seeds grading. In (Agustin and Oh, 2008), the authors focused on the quality control of grain of rice, 
regarding different criteria of shape and color. From these criteria, a classification method based on neural network is used to qualify each grain. This approach gives good results for the classification of complete, broken and colors defect rice grain. However this method is applied on images with separated grain. The segmentation issue is then simplified by an operator or a mechanical system (vibrating bowl or slot) to separate the grain in front of the camera.

Other studies have been conducted on the cereal segmentation topic, mainly on wheat and rice. The researches in (Yao et al., 2010) and (Faessel and Courtois, 2011) focused on detection and separation of rice grain. They both address the problem by working on a binary image obtained by a threshold to separate the objects from the background. In (Yao et al., 2010), the authors then work on the contours and search the concaves angles to connect them two at a time in order to detect objects boundaries. In (Faessel and Courtois, 2011), the authors used a mathematical morphology method on the binary image: a skeleton operation on the background. The open lines of the skeleton, without ending, are then combined under some constraints to obtain the objects boundaries. These two methods give good results on image of touching grain with low density of objects. The computation times are short, but these methods are not adapted for images with heaps and high density of seeds.

\section{ACQUISITION SYSTEM}

The acquisitions are made in a cabin (Figure 1) which integrates a camera and a lighting system. This cabin offers stable and reproducible acquisition condition, independently from the external lighting.

Some improvements have been made on the existing system available at the industrial partner. The lighting system and the camera have been replaced by new material. The aim was to improve the quality and the stability of the color image acquisition. The lighting source retained is composed of white LEDs. These LEDs have a continuous spectrum in the visible range and were chosen for their stability over time in term of luminous intensity. As LEDs are punctual sources, a diffuser is placed downstream to ensure the lighting homogeneity in the acquisition area. The image acquisition is performed at a distance of $400 \mathrm{~mm}$ from the object plan by a CMOS mono sensor color camera of 5 megapixel with a $5 \mathrm{~mm}$ lens. The chosen camera was a Basler acA2500-14gc. It offers a resolution on the object plan around 6 pixels per millimeter, which is important for our application as the objects have a size of only few millimeters.

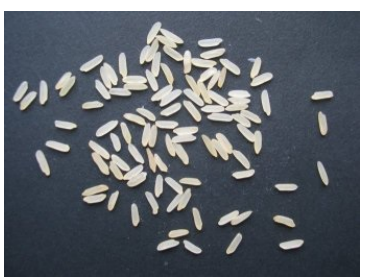

(a) Original image.

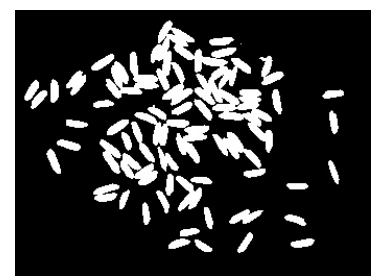

(b) Binarized image.
Figure 2: Binarization with Otsu's method.

The image acquisitions presented in this paper were obtained with this system.

\section{SHAPE LEARNING}

A binary image can be obtained in many classical different ways. For example, with rice seeds which are well contrasted with a black background, Otsu's method (Otsu, 1979) can be applied, see Figure 2.

A background learning without any seed then subtraction could also be considered. In difficult cases, it is possible for an human operator to choose manually correct thresholds for gray level images or color images. He could also separate some pixels in two classes and let a k-means clustering algorithm do the rest (MacQueen, 1967). The binarization could also be made using the image gradient.

From a binarized image of isolated seeds, the following features of each seed are extracted:

Area. It is the number of pixels inside the seed.

Eccentricity. It is a scalar, between 0 and 1, which specifies the eccentricity of the ellipse that has the same second-moments as the region. The eccentricity is the ratio of the distance between the foci of the ellipse and its major axis length. An ellipse whose eccentricity is 0 is actually a circle, while an ellipse whose eccentricity is 1 is a line segment.

Major axis length. It is the distance between the end points of the longest line that could be drawn through the seed. The major axis endpoints are found by computing the pixel distance between every combination of border pixels in the seed boundary and finding the pair with the maximum length.

Minor axis length. It is the distance between the end points of the longest line that could be drawn through the seed while maintaining perpendicularity with the major axis.

Perimeter. It is the number of pixels of the boundary. Roundness. It is a measure of how closely the shape of an object approaches that of a circle. It is equalled to $4 \pi$ times the area divided by the square of the perimeter. 


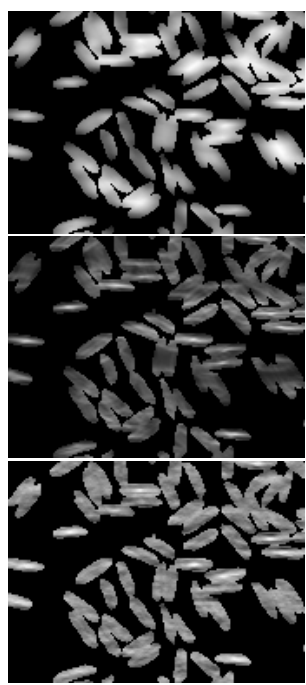

$\theta=0$

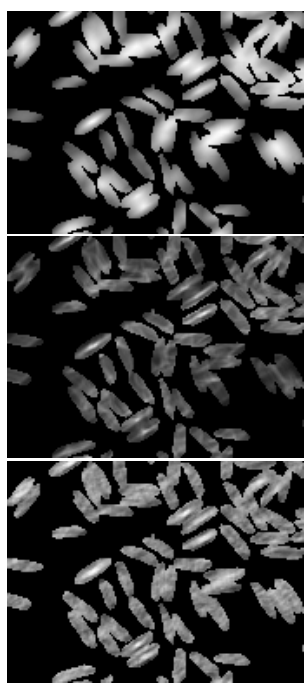

$\theta=\pi / 5$

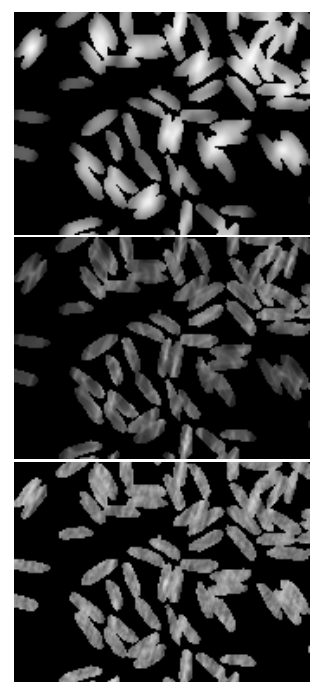

$\theta=2 \pi / 5$

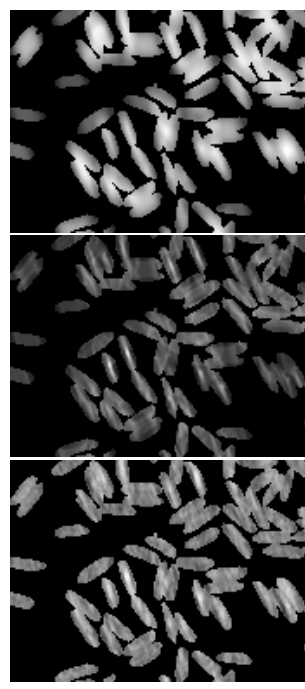

$\theta=3 \pi / 5$

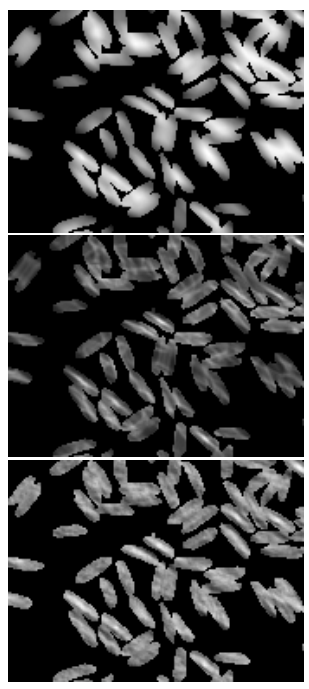

$\theta=4 \pi / 5$

Figure 3: From top to bottom, examples of $E_{1}, E_{2}$ and $E_{3}$ computed with the same ellipse and five different orientations.

In the learning process, a k-means clustering algorithm on the statistics allows to separate the seeds in three categories: broken, isolated and adjacent. The broken or adjacent grain kernels can be present or absent. The category with the adjacent ones is not taken into account during the learning process.

\section{SEGMENTATION}

\subsection{Energy maps}

From the image, three kind of energy maps can be extracted based on the matter $E_{1}$, the gradient gap on the boundary $E_{2}$ and the normal to the boundary $E_{3}$. They take their values between $[0,1]$. Let $\Omega$ be the image domain, $\omega$ be the shape to test and $x \in \Omega$ a location. They are defined as follows:

$$
E_{1}(\omega)=\frac{1}{\operatorname{area}(\omega)} \int_{\omega} u_{b}(x) \mathrm{d} x
$$

where area is a function which gives the area of the shape, $u_{b}$ denotes the binarized image with 1 if a pixel is belong to a seed and 0 otherwise,

$$
E_{2}(\omega)=\frac{1}{\max _{y \in \Omega}(\|\nabla u(y)\|)} \frac{1}{|\partial \omega|} \int_{\partial \omega}\|\nabla u(x)\| \mathrm{d} x
$$

where $\nabla u(x)$ denotes the image gradient, $|\partial \omega|$ denotes the length of the object boundary and $\|$.$\| is the Eu-$ clidean norm,
$E_{3}(\omega)=\frac{1}{2|\partial \omega|} \int_{\partial \omega}\left\langle\frac{\nabla u(x)}{\sqrt{|\nabla u(x)|^{2}+\varepsilon^{2}}}, n(x)\right\rangle \mathrm{d} x+\frac{1}{2}$

where $\langle.,$.$\rangle denotes the Euclidean scalar product, n(x)$ denotes the outward normal to $\omega$ at location $x \in \partial \omega$ and $\varepsilon$ is a regularization parameter that discard faint transitions.

The function $E_{1}$ checks the matter quantity inside the considered shape, but it baldly reacts inside adjacent seeds or bigger seed. The function $E_{2}$ reacts well for the boundaries between the background and the seeds, but not between two seeds. The function $E_{3}$ is working with high and low contrasts, but it could suffered from shading issues. Therefore the three energies are used together in order to avoid the disadvantages and combine the advantages (see Section 5.3)

The behaviour of the three energy maps are illustrated in Figure 3. The ellipse had been chosen in the case of rice seeds. Each figures present five orientation maps obtained from a zoom of the input image in Figure 2. These energy maps are more efficient with isolated seeds and on the boundary of seed heaps. For this reason the segmentation algorithm attacks the heaps progressively from their boundaries to their cores.

The computation of the energy maps is quite simple and fast. Firstly a filter corresponding to the operation is built. Secondly a simple convolution with the image is performed. The speed comes from the fact that Fourier transform of a convolution is the pointwise product of Fourier transforms. 


\subsection{Active contour}

The used objects to create the energy maps have only simple shapes like ellipses with a limited range of axes sizes. The computational time is the main reason. But to accurately detect every object in the image, we need to obtain the most precise boundaries.

As the objects to detect have a generical shape, we decided to use the method based on an active contour with a geometric shape prior proposed in (Bresson et al., 2006). This method follows the well-known energy functional model presented in (Chen et al., 2002) where the shape prior given in (Leventon et al., 2000) is integrated. Finally, to improve the robustness of the method, Bresson et al. add a region-based energy term based on the Mumford-Shah functional (Chan and Vese, 2001). This method is then based on three complementary terms dedicated to shape, boundary and region inside the contour.

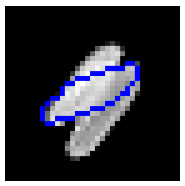

(a) Bad start

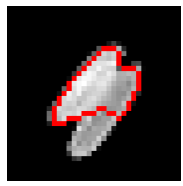

(b) Rejection

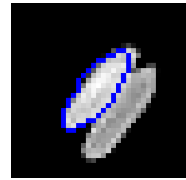

(c) Good start

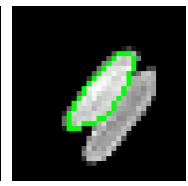

(d) Acceptance
Figure 4: Behaviour of active contour.

The starting contour is the ellipse that minimizes the previous energy maps, see the next section for more details. Figure 4 illustrates the behavior of the active contour in the cases of bad starting contour (a) and a good one (c). The starting contours are represented in blue. The first leads to a final contour in red with a shape distant from an ellipse and is rejected. The second leads to a final contour in green with a shape similar to an ellipse and is accepted.

\subsection{Algorithm}

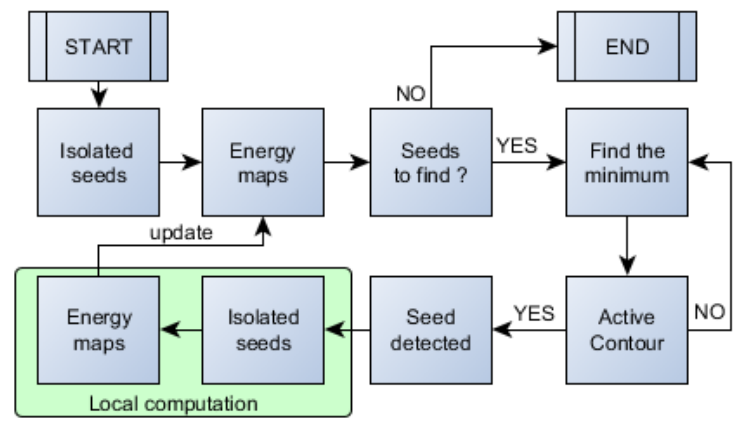

Figure 5: Scheme of the algorithm.

From a binary image, the proposed deterministic algorithm detects the isolated seed, by comparing the shape of each connex set to known criteria. For example on rice seeds, the area, the perimeter and the roundness are compared to the results of shape learning. If they match, the connex set is considered as a seed and is not anymore used in the process.

In this paper, the studied seeds are well approximated with ellipses. The algorithm compute three energy maps with a range of parameters: $\theta$ the orientation, $a$ the major axis length and $b$ the minor length axis. It sorts the values of the following function:

$$
E=\sqrt{E_{1}^{2}+E_{2}^{2}+E_{3}^{2}}
$$

into a vector $\phi$. At the minimum, the active contour step is launched. If its result is rejected, the algorithm looks for the next value in $\phi$ until it accepts the detection and considers that it detects a seed. When a seed is detected, a black shape replaced it in the image. The algorithm checks the connex sets in the local zone. If there are considered as a seed and there are not anymore used in the process. Then local energy maps are computed in the neighborhood in order to update the energy maps and update $\phi$. The process is iterated until there are no more pixels considered as belonging to a seed or if the algorithm is unable to explain the final connex sets with seeds.

\section{NUMERICAL RESULTS}

\subsection{Numerical results on shape learning}

Figure 6 presents some seeds. From left to right, there are rice, pea, barley and wheat. The images are segmented and statistics on the features of isolated seeds are computed. Table 1 presents these statistics which will be helpful for the parameter selection in the proposed segmentation algorithm.

\subsection{Numerical results on segmentation}

Figures 7, 8, 9 and 10 present the segmentation results of the proposed algorithm with different kinds of seeds. Figures 7 and 8 are both on rice seeds but the second one has a higher density. Results on pea and barley seeds are presented in respectively Figures 9 and 10. Thanks to the results from Table 1, the chosen shape parameters generate circular to elongated ellipses. For example, pea seeds are approximated by ellipses, more circular than the two others.

Most of the seeds are well segmented. The best result is obtained with the pea seeds because there is less contact between the seeds. The use of superellipses, also known as Lamé curves, could improve the barley seeds which are more pointed. 
Table 1: Results from shape learning with isolated seeds based on the images presented in Figure 6.

\begin{tabular}{|c|c|c|c|c|c|c|}
\hline \multicolumn{7}{|c|}{ Rice seeds, Figure 6 (a) } \\
\hline Features & Area & Major axis length & Minor axis length & Perimeter & Eccentricity & Roundness \\
\hline Minimum & 218 & 23 & 10 & 61 & 0.804 & 0.470 \\
\hline Maximum & 559 & 51 & 15 & 114 & 0.972 & 0.840 \\
\hline Mean & 422 & 43 & 13 & 96 & 0.950 & 0.576 \\
\hline Median & 430 & 44 & 13 & 98 & 0.957 & 0.560 \\
\hline Standard deviation & 64 & 6 & 1 & 10 & 0.026 & 0.067 \\
\hline \multicolumn{7}{|c|}{ Pea seeds, Figure 6 (b) } \\
\hline Features & Area & Major axis length & Minor axis length & Perimeter & Eccentricity & Roundness \\
\hline Minimum & 894 & 35 & 29 & 115 & 0.208 & 0.601 \\
\hline Maximum & 2061 & 55 & 48 & 176 & 0.738 & 0.927 \\
\hline Mean & 1322 & 43 & 39 & 140 & 0.427 & 0.845 \\
\hline Median & 1283 & 43 & 39 & 138 & 0.434 & 0.850 \\
\hline Standard deviation & 233 & 4 & 3 & 12 & 0.108 & 0.045 \\
\hline \multicolumn{7}{|c|}{ Barley seeds, Figure 6 (c) } \\
\hline Features & Area & Major axis length & Minor axis length & Perimeter & Eccentricity & Roundness \\
\hline Minimum & 842 & 61 & 16 & 149 & 0.952 & 0.309 \\
\hline Maximum & 1812 & 101 & 24 & 253 & 0.979 & 0.528 \\
\hline Mean & 1312 & 82 & 20 & 192 & 0.968 & 0.442 \\
\hline Median & 1377 & 82 & 21 & 191 & 0.968 & 0.448 \\
\hline Standard deviation & 266 & 11 & 2 & 25 & 0.006 & 0.045 \\
\hline
\end{tabular}

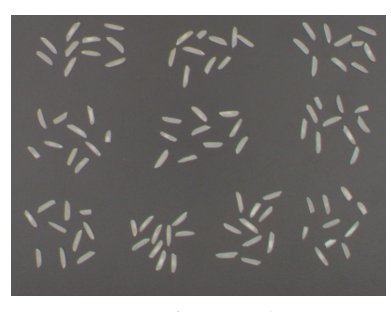

(a) Rice seeds

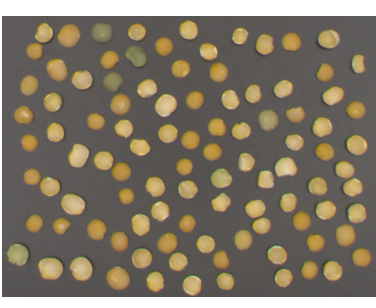

(b) Pea seeds

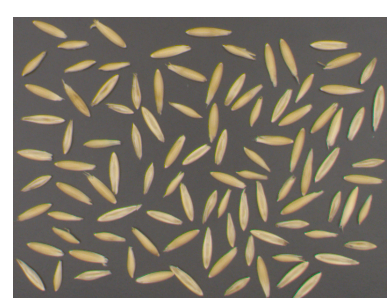

(c) Barley seeds

Figure 6: Isolated seeds used for shape learning.

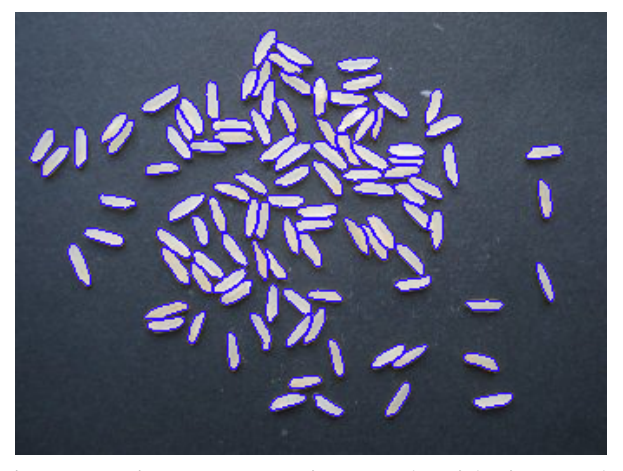

Figure 7: First segmentation result with rice seeds.

\section{CONCLUSION}

This paper proposes a deterministic approach to perform the visual quality control of cereal seeds samples. This operation called visual grading can be treated in three steps: acquisition, segmentation and classification. An acquisition system of color images has been created to collect the data. A new segmentation approach has been developed. The proposed

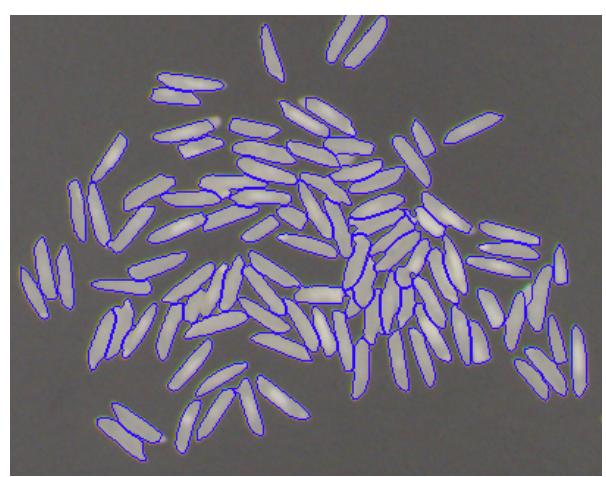

Figure 8: Second segmentation result with rice seeds.

method has two steps. The first one uses the region, the edges and the normals to the boundary. In order to be accurate and avoid human parameter configuration, it is necessary to learn shape information on isolated seeds. The second step is based on active contours. The preliminary results on rice, pea and barley seeds are promising.

Experiments with higher density and with other 


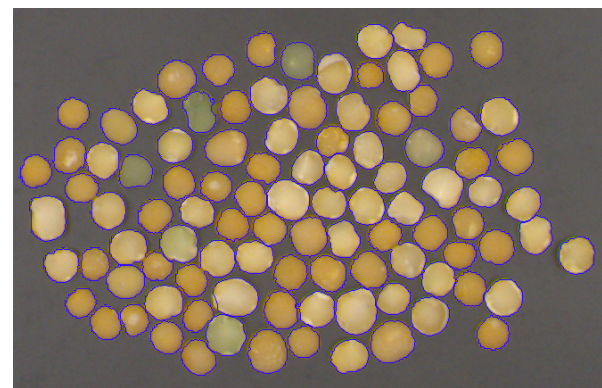

Figure 9: Segmentation result with pea seeds.

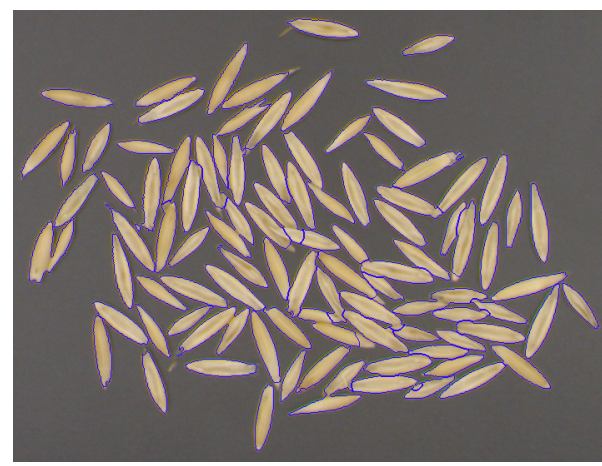

Figure 10: Segmentation result with barley seeds.

type of seeds or objects are in progress. So far, only ellipses have been used for the shape, in order to be more accurate, superellipses and ovals will also be considered. Other tests like comparison with human operator and reproducibility on the same sample in different configurations are also underway. Our approach is deterministic, in parallel, we are building a probabilistic one (Dubosclard et al., 2014) based on marked point processes (Descombes et al., 2009) and the same step of active contour with shape prior. A comparative study between the two approaches is in progress. The integration of a 3D data acquisition system like stereovision with two cameras is under investigation. Despite the hardware cost, such data might be useful in particular to enrich the birth map but also to provide criteria for the classification stage.

\section{Acknowledgement}

This CIFRE thesis work was made possible thanks to the involment of Alpha MOS company.

\section{REFERENCES}

Agustin, O. and Oh, B.-J. (2008). Automatic milled rice quality analysis. In Second International
Conference on Future Generation Communication and Networking, 2008. FGCN '08, volume 2, pages 112-115.

Bresson, X., Vandergheynst, P., and Thiran, J.-P. (2006). A variational model for object segmentation using boundary information and shape prior driven by the mumford-shah functional. International Journal of Computer Vision, 68(2):145162.

Chan, T. and Vese, L. (2001). Active contours without edges. IEEE Transactions on Image Processing, 10(2):266-277.

Chen, Y., Tagare, H., Thiruvenkadam, S., Huang, F., Wilson, D., Gopinath, K., Briggs, R., and Geiser, E. (2002). Using prior shapes in geometric active contours in a variational framework. International Journal of Computer Vision, 50(3):315328.

Descombes, X., Minlos, R., and Zhizhina, E. (2009). Object extraction using a stochastic birth-anddeath dynamics in continuum. Journal of Mathematical Imaging and Vision, 33:136-139.

Dubosclard, P., Larnier, S., Konik, H., Herbulot, A., and Devy, M. (2014). Automatic method for visual grading of seed food products. In International Conference on Image Analysis and Recognition (ICIAR), volume 1 of Lecture Notes in Computer Science, pages 485-495.

Faessel, M. and Courtois, F. (2011). Touching grain kernels separation by gap-filling. Image Analysis and Stereology, 28(3):195-203.

Leventon, M., Grimson, W. E. L., and Faugeras, O. (2000). Statistical shape influence in geodesic active contours. In IEEE Conference on Computer Vision and Pattern Recognition, 2000, volume 1, pages 316-323.

MacQueen, J. B. (1967). Some methods for classification and analysis of multivariate observations. In Cam, L. M. L. and Neyman, J., editors, Proc. of the fifth Berkeley Symposium on Mathematical Statistics and Probability, volume 1, pages 281-297. University of California Press

Otsu, N. (1979). A threshold selection method from gray-level histograms. IEEE Transactions on Systems, Man and Cybernetics, 9(1):62-66.

Yao, Q., Zhou, Y., and Wang, J. (2010). An automatic segmentation algorithm for touching rice grains images. In International Conference on $\mathrm{Au}$ dio Language and Image Processing (ICALIP), pages 802-805. 